\title{
The effect of whole body vibration on the H-reflex, the stretch reflex, and the short-latency response during hopping
}

\author{
R. Ritzmann ${ }^{1}$, A. Kramer ${ }^{1}$, A. Gollhofer ${ }^{1}$, W. Taube ${ }^{2}$ \\ ${ }^{1}$ Institute of Sport and Sport Science, University of Freiburg, Freiburg, Germany, ${ }^{2}$ Department of Medicine, Movement and Sport \\ Science, University of Fribourg, Fribourg, Switzerland \\ Corresponding author: Ramona Ritzmann, MA, Institute of Sport and Sport Science, University of Freiburg, Schwarzwaldstraße 175, \\ 79117 Freiburg, Germany. Tel: +49 761203 4557, Fax: +49 761203 4534, E-mail: ramona.ritzmann@sport.uni-freiburg.de
}

\begin{abstract}
The effect of whole body vibration (WBV) on reflex responses is controversially discussed in the literature. In this study, three different modalities of reflex activation with increased motor complexity have been selected to clarify the effects of acute WBV on reflex activation: (1) the electrically evoked H-reflex, (2) the mechanically elicited stretch reflex, and (3) the shortlatency response (SLR) during hopping. WBV-induced changes of the H-reflex, the stretch reflex, and the SLR during hopping were recorded in the soleus and gastrocnemius muscles and were analyzed before, during (only the H-reflex), immediately after, $5 \mathrm{~min}$ and $10 \mathrm{~min}$ after $\mathrm{WBV}$. The main findings were that
\end{abstract}

(1) the H-reflexes were significantly reduced during and at least up to $5 \mathrm{~min}$ after WBV, (2) the stretch reflex amplitudes were also significantly reduced immediately after WBV but recovered to their initial amplitudes within $5 \mathrm{~min}$, and (3) the SLR during hopping showed no vibration-induced modulation. With regard to the modalities with low motor complexities, the decreased $\mathrm{H}$ - and stretch reflex responses are assumed to point toward a reduced Ia afferent transmission during and after WBV. However, it is assumed that during hopping, the suppression of reflex sensitivity is compensated by facilitatory mechanisms in this complex motor task.
Performance improvements in response to whole body vibration (WBV) are controversially discussed in the literature (Nordlund \& Thorstensson, 2007; Rittweger, 2010). Several reports state that WBV can have a beneficial effect on strength (Delecluse et al., 2003; Roelants et al., 2004) and power (Roelants et al., 2004; Rees et al., 2008). These improvements were speculated to be associated with an enhanced neural excitation (Cochrane \& Stannard, 2005; Cardinale \& Bosco, 2003; Rittweger et al., 2003), possibly achieved by increased reflex activation (Bosco et al., 2000; Cardinale \& Bosco, 2003; Cochrane \& Stannard, 2005). In contrast, there are numerous studies that could not find any WBV-induced performance improvements (Rittweger et al., 2000; de Ruiter et al., 2003), and some authors questioned any additional effects of WBV if standardized training protocols are compared with and without WBV (Nordlund \& Thorstensson, 2007). Thereby, some authors proposed that the neural facilitation lacks validity, and WBV is more likely to induce an inhibition rather than a facilitation (Nordlund \& Thorstensson, 2007).

Despite its widespread use in different areas of rehabilitative sports medicine, geriatrics and as a training method for elite athletes (for review, see Rittweger, 2010) and despite the substantial amount of related arti- cles, the underlying neuromuscular mechanisms in response to WBV are poorly understood. A better understanding of the mechanisms is a prerequisite for a specific and substantiated application of WBV training. So far, there are numerous articles that document the neurophysiological effects in response to isolated vibration applied to the muscle belly or the tendon (Arcangel et al., 1971; Burke et al., 1976), but it has yet to be shown whether these results can be transferred to WBV. Isolated muscle or tendon vibration activates primary and secondary muscle spindle endings as well as the $\mathrm{Ib}$ afferents from Golgi tendon organs (Burke et al., 1976). This activation of muscle spindle endings is thought to elicit a succession of stretch reflexes, the "tonic vibration reflex" (Matthews, 1966). It has been shown that isolated muscle and tendon vibrations reduce Ia afferent transmission (Arcangel et al., 1971; van Boxtel, 1986), probably mainly due to an increased level of presynaptic inhibition (PSI; Gillies et al., 1969).

Concerning the effect of isolated muscle or tendon vibrations mentioned above, it might be hypothesized that WBV also reduces spinal reflex responses. However, the reported reflex adaptations following WBV are inconsistent in the literature (Rittweger, 2010). For instance, some authors demonstrated an inhibition of the 
soleus (SOL) H-reflex (Armstrong et al., 2008; Sayenko et al., 2010) and stretch reflex responses (Roll et al., 1980) after WBV. Those results are well in line with the findings observed after locally applied muscle and tendon vibration. However, other authors reported facilitated reflexes after WBV (Rittweger et al., 2003; Melnyk et al., 2008), which were argued to be beneficial for performance-related parameters like increases in jump height (Bosco et al., 2000; Cochrane \& Stannard, 2005). In light of the articles of McBride et al. (2010) and Hopkins et al. (2009), who did not observe any differences in response to acute WBV exposure at all, drawing functional conclusions has become even more delicate. To explain at least part of the contradictory results, it has been proposed that electrically evoked H-reflex and mechanically elicited stretch reflex responses might be differently affected by WBV (Rittweger, 2010). Furthermore, the time interval between WBV treatment and reflex measurement may influence the outcome, as it was shown in studies applying local muscle and tendon vibrations (Arcangel et al., 1971).

Therefore, this study investigated the influence of WBV on different modalities of reflex activation with increased motor complexity at distinct time intervals during and after the WBV treatment: (1) the electrically elicited H-reflex, (2) the mechanically evoked stretch reflex, and (3) the short-latency response (SLR) during hopping. The rationale behind testing $\mathrm{H}$-reflexes and stretch reflexes was their different point of origin: $\mathrm{H}$-reflexes bypass the muscle spindles, whereas stretch reflexes are largely dependent on the sensitivity of the muscle spindles and the $\alpha-\gamma$ linkage. It is well-known that the amplitude of the H-reflex is independent of the activity of the $\gamma$-motoneurons, whereas the stretch reflex can be modulated by the fusimotor drive (Granit, 1975; Hagbarth \& Macefield, 1995). Furthermore, it was demonstrated that the amplitude of the H-reflex is strongly more affected by changes in PSI than the stretch reflex (Morita et al., 1998). In the third experimental condition, we investigated the modulation of reflex contributions in hopping to assess the effects of WBV on stretch reflex components in a functional task. In all three conditions, reflex responses were measured at distinct time intervals during and after the WBV treatment to highlight the time dependency of WBV-induced reflex modulation.

We hypothesized that according to the effects observed with locally applied muscle and tendon vibration and based on the data we collected in a pilot study, WBV would reduce the H-reflex, the stretch reflex, and the SLR during hopping and that those effects would decline over time.

\section{Materials and methods}

Subjects

Twenty-two subjects ( 12 females and 10 males, age $26 \pm 3$ years) volunteered to participate in this study. All subjects gave written informed consent to the experimental procedure, which was approved by the ethics committee of the University of Freiburg and was in accordance with the latest revision of the Declaration of Helsinki. The subjects were healthy with no previous neurological irregularities or injuries of the lower extremity.

\section{Experimental design}

A single-group repeated-measures study design was used to evaluate acute WBV-induced effects on reflex responses. For that purpose, three different protocols were used: in the first protocol, we investigated the influence of WBV on the Ia afferent transmission by means of H-reflex measurements. The second protocol aimed to assess vibration-induced effects on the mechanically evoked stretch reflex. The third protocol quantified the influence of WBV on the stretch reflex contribution during hopping. Reflex responses for each protocol were recorded at a minimum of four different time intervals: just before vibration $\left(t_{1}\right)$, immediately after $\left(t_{3}\right), 5 \min \left(t_{4}\right)$ and 10 min after vibration $\left(t_{5}\right)$. In protocol 1 , $\mathrm{H}$-reflexes were additionally recorded during WBV $\left(\mathrm{t}_{2}\right)$. Recording of stretch reflexes and the SLR of hopping during WBV was not feasible from a methodological point of view. In protocol 2 , stretch reflex responses were recorded twice $\left(t_{0}\right.$ and $t_{1}$ ) before the WBV intervention was started to control the reproducibility of mechanically evoked stretch reflex responses. The protocols were conducted on separate days with a minimum of 1 day rest in between.

\section{WBV}

The WBV device was a side-alternating vibration platform (Galileo Sport, Novotec Medical, Pforzheim, Germany), which generates vibration by platform oscillations along the sagittal axis. In this study, the axis of rotation was placed in between the subjects' feet, and the feet were placed $21 \mathrm{~cm}$ away from the axis of rotation resulting in a vibration amplitude of $4 \mathrm{~mm}$. The vibration frequency was set to $22 \mathrm{~Hz}$. During those $60 \mathrm{~s}$, the subjects were exposed to vibrations, and the subjects maintained a static body position with a knee angle of $30^{\circ}$ and a forefoot stance. The subjects were instructed to place their hands on their hips, direct their head and eyes forward, and distribute their weight equally on both feet.

\section{Electromyographic (EMG) recording}

Bipolar $\mathrm{Ag} / \mathrm{AgCl}$ surface electrodes (Ambu Blue Sensor P, Ballerup, Denmark; diameter $9 \mathrm{~mm}$, center-to-center distance $34 \mathrm{~mm}$ ) were placed over the Musculus (M.) SOL, the M. gastrocnemius medialis (GM), and the M. tibialis anterior (TA) of the right leg. The longitudinal axes of the electrodes were in line with the direction of the underlying muscle fibers. The reference electrode was placed on the patella. Interelectrode resistance was kept below $5 \mathrm{k} \Omega$ by means of shaving, light abrasion, degreasing, and disinfection of the skin. The (EMG) signals were transmitted to the amplifier (band-pass filter from $10 \mathrm{~Hz}$ to $1 \mathrm{kHz}, 1000 \times 1000$ amplified) via shielded cables and recorded with $4 \mathrm{kHz}$. The cables were carefully taped to the skin.

\section{Protocol 1: H-reflex}

Protocol 1 was conducted with 22 subjects. Modulation in Ia afferent transmission of the SOL in response to WBV was assessed by H-reflex measurements; H-reflexes were elicited by peripheral nerve stimulation (PNS). For the PNS, an electrical stimulator (Alea Solutions ${ }^{\circledR}$ type AS100, Zurich, Switzerland) was used, generating single rectangular pulses of 1-ms duration. The cathode $(2 \mathrm{~cm}$ in diameter) was placed in the popliteal fossa and moved until the best position was found for eliciting an H-reflex in the 


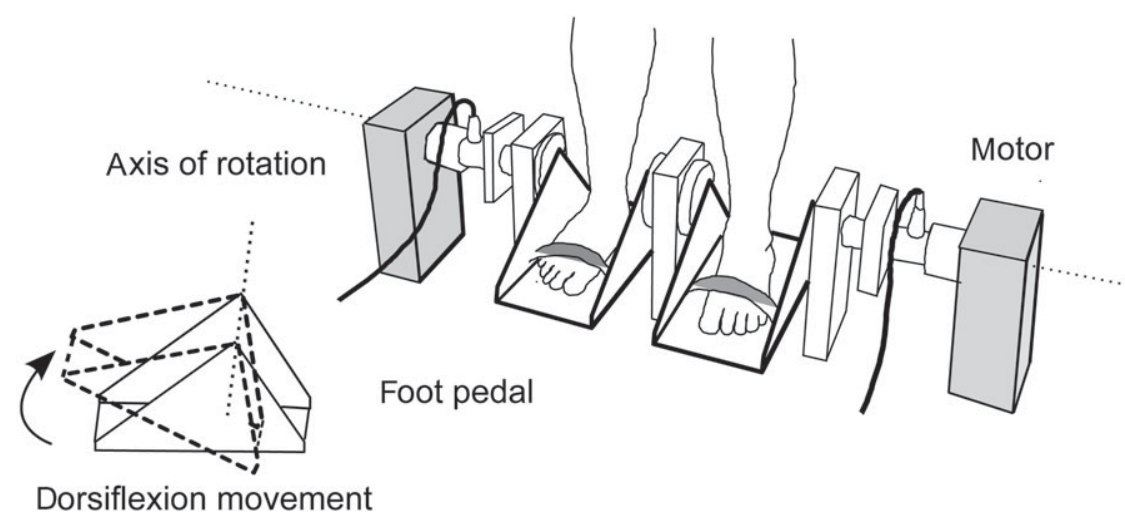

Fig. 1. Illustration of the ankle ergometer used to evoke stretch reflex responses in the soleus and gastrocnemius medialis muscles. The stretch is induced by an ankle dorsiflexion, which in turn is forced by the upward movement of the foot pedal.

SOL. The anode $(10 \times 5 \mathrm{~cm}$ dispersal pad $)$ was fixed directly below the patella on the anterior aspect of the knee. H-reflexes were elicited by electrically stimulating the posterior tibial nerve with an interstimulus interval of $4 \mathrm{~s}$. Throughout each of the defined time intervals $\left(t_{1}, t_{2}, t_{3}, t_{4}\right.$, and $\left.t_{5}\right)$, an $H / M$ recruitment curve was recorded with the subjects standing in an upright position. For that purpose, the stimulation current was successively augmented, ranging from subthreshold stimulation intensities to intensities sufficient to elicit H-reflexes to supramaximal intensities for the maximal M-wave (Taube et al., 2008).

\section{Protocol 2: Stretch reflex}

In 13 subjects, passive dorsiflexions generated by a custom-made ankle ergometer evoked stretch reflex responses in SOL and in GM. The ankle ergometer was driven by two independent motors that controlled the rotation of the right and the left foot pedal. The participants were fixed by a strap-binding system with their feet resting on the rotation platform of the ergometer. The rotation axis of the upper ankle joint coincided with the rotation axis of the platform. A mechanically induced dorsiflexion movement at the ankle joint with an amplitude of $6^{\circ}$ and a velocity of $150^{\circ} / \mathrm{s}$ evoked a stretch and hence a reflex response in the triceps surae muscle, which occurred depending on the subject with an interindividual latency of 35-55 ms (Fig. 1). Dorsiflexions were carried out in two different modalities: first, in a sitting position $\left(90^{\circ}\right.$ hip, knee, and ankle joint angle) to evoke stretch reflex responses in relaxed muscles and second, just like in the H-reflex measurement in an upright standing position, in which the muscles are slightly activated to ensure postural equilibrium (Hayashi et al., 1992). At each of the defined time intervals $\left(t_{0}, t_{1}, t_{3}, t_{4}\right.$, and $\left.t_{5}\right), 20$ stretch reflexes with an interstimulus interval of $4 \mathrm{~s}$ were evoked.

\section{Protocol 3: SLR in hopping}

Twenty subjects performed stiff hopping, i.e. hopping with a small range of motion in the hip, knee, and ankle joint and short groundcontact times (GCT). At each of the defined time intervals $\left(t_{1}, t_{3}, t_{4}\right.$, and $t_{5}$ ), 40 jumps were performed on a force platform (Kistler ${ }^{\circledR}$, Wintherthur, Switzerland) to determine the ground reaction forces (GRF) and the corresponding neuromuscular activity. While jumping, the subjects kept their hands akimbo to avoid supportive movement of the arms.

\section{Data processing}

Peak-to-peak amplitudes of the H-reflexes and $\mathrm{M}$-waves were calculated, and the maximal H-reflex $\left(\mathrm{H}_{\max }\right)$ was expressed relative to $\left(\mathrm{M}_{\max }\right)\left(\mathrm{H}_{\max } / \mathrm{M}_{\max }\right.$ ratio). Peak-to-peak amplitudes of the stretch reflex responses were calculated, averaged, and subsequently normalized to $t_{1}$ for each time interval. The stretch reflex time interval was defined as the interval from the initial deflection of the EMG signal from the baseline to the second crossing of the baseline.

For hopping, the EMG was rectified, averaged, and integrated (iEMG). The iEMG of the preactivation phase (150-0 ms before ground contact) and the SLR (30-60 ms after ground contact) were calculated (Hobara et al., 2007) and normalized to $t_{1}$. The GRF were used to determine the rate of force development (RFD), the maximal force $\left(\mathrm{F}_{\max }\right)$, and the GCT.

\section{Statistic}

To test for reflex changes over time $\left(\mathrm{t}_{0}-\mathrm{t}_{5}\right)$ in protocol 1 (H-reflex), protocol 2 (stretch reflex), and protocol 3 (SLR during hopping), a repeated measures analysis of variance was used. A Bonferroni post-hoc test was used to correct for multiple testing. The level of significance was set to $P \leq 0.05$, and statistically significant differences were marked with a symbol $(*)$. To make sure that particular parameters $\left(\mathrm{M}_{\max }, \mathrm{F}_{\max }, \mathrm{RFD}, \mathrm{GCT}\right.$, jump height, the iEMG of TA in the phase of the SLR, and the preactivation in SOL, GM, and TA) did not change over time in response to WBV, equivalence statistics were used (for that purpose, the $95 \%$ confidence intervals were calculated for the differences between the corresponding values in $t_{1}$ and $t_{2}\left(t_{3}, t_{4}\right.$, and $t_{5}$, respectively). The acceptable bounds were determined based on the differences observed in a repeated measurement without intervention (Borman et al., 2009). If the confidence interval stayed within those bounds, the differences were statistically equal. In case of statistical equivalence, the respective parameter is marked with a symbol $(\approx)$. All statistical analysis was conducted using the SPSS 16.0 software (SPSS Inc., Chicago, Illinois, USA). All data are presented as group mean values \pm standard deviation unless otherwise stated.

\section{Results}

H-reflex

The $\mathrm{H} / \mathrm{M}$ recruitment curves and the corresponding $\mathrm{H}_{\max } /$ $\mathrm{M}_{\max }$ ratios of one representative subject are shown in Figure 2. The individual data as well as the mean data of the group (Fig. 3) highlight that the $H_{\max } / M_{\max }$ ratios in SOL were highly reduced during WBV, followed by a phase of recovery within $10 \mathrm{~min}$ after WBV $\left(\mathrm{t}_{1}: 0.55 \pm 0.19 ; \mathrm{t}_{2}: 0.27 \pm 0.14 ; \mathrm{t}_{3}: 0.48 \pm 0.14 ; \mathrm{t}_{4}:\right.$ $0.48 \pm 0.16$; and $t_{5}: 0.50 \pm 0.16, P<0.05$; Fig. 3$)$. The 

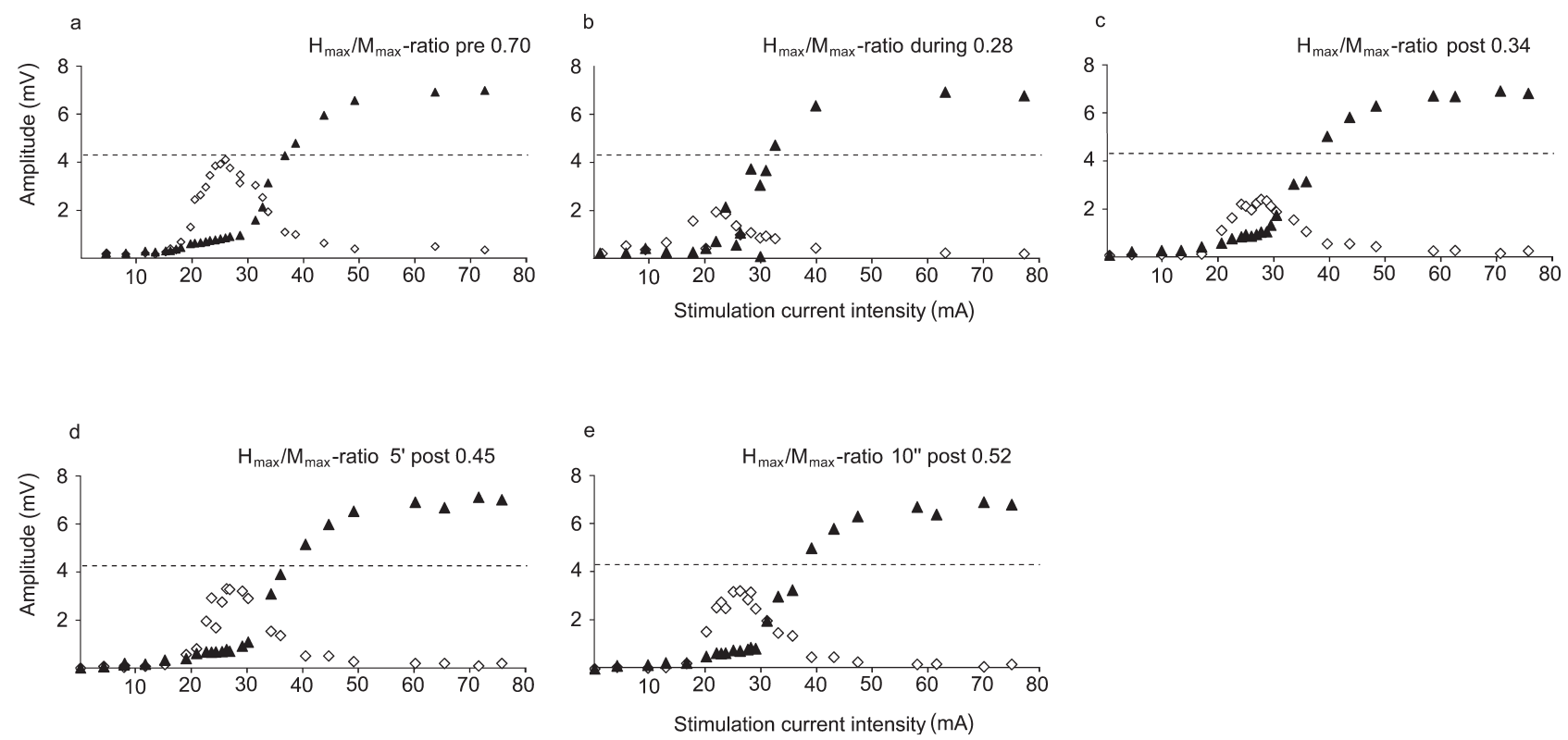

Fig. 2. Modulation of soleus H-reflexes in response to acute WBV in one representative subject: H/M recruitment curves $(\diamond$ H-reflexes, $\boldsymbol{\Delta}$ M-waves) and the resulting $\mathrm{H}_{\max } / \mathrm{M}_{\max }$ ratios recorded before (a), during (b), immediately after (c), $5 \mathrm{~min}$ (d), and $10 \mathrm{~min}$ (e) after WBV treatment. The maximal M-wave $\left(\mathrm{M}_{\max }\right)$ was of similar size before (a), during (b), and after WBV (c, d, and e), whereas the maximal H-reflex $\left(\mathrm{H}_{\max }\right)$ was highly reduced during WBV and successively recovered up to 10 min after WBV. The horizontal dashed line marks the initial value of $\mathrm{H}_{\max }$ recorded in the control condition (a) before WBV.

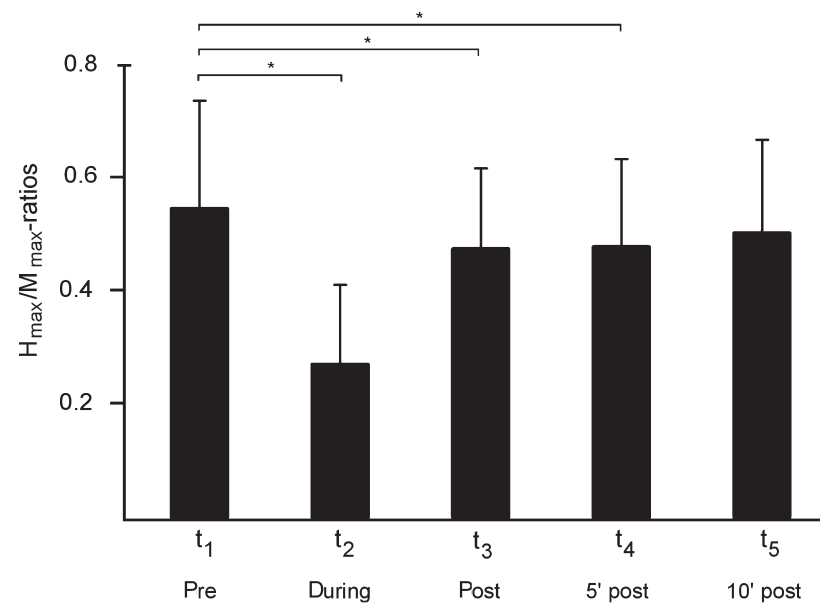

Fig. 3. Grand mean of the $H_{\max } / \mathrm{M}_{\max }$ ratios of the soleus muscle $(n=22)$ at the different time intervals before $\left(\mathrm{t}_{1}\right)$, during $\left(\mathrm{t}_{2}\right)$, immediately after $\left(\mathrm{t}_{3}\right), 5 \mathrm{~min}$ and $10 \mathrm{~min}$ after vibration treatment $\left(t_{4}\right.$ and $\left.t_{5}\right)$.

$\mathrm{H}_{\max } / \mathrm{M}_{\max }$ ratios were significantly decreased at the time intervals during $\left(\mathrm{t}_{2}: P<0.05\right)$ and within 5 min after WBV ( $\left.\mathrm{t}_{3}: P<0.05 ; \mathrm{t}_{4}: P<0.05\right)$ compared with control $\mathrm{H}_{\max } / \mathrm{M}_{\max }$ ratios established during the control condition before WBV (Figs 2 and 3). During the entire measurement, $\mathrm{M}_{\max }$ was statistically equal $\left(\mathrm{t}_{1}: 7.1 \pm 2.7 ; \mathrm{t}_{2}\right.$ : $7.4 \pm 2.6 \approx ; \mathrm{t}_{3}: 7.5 \pm 2.4 \approx ; \mathrm{t}_{4}: 7.2 \pm 2.3 \approx ;$ and $\mathrm{t}_{5}$ : $7.2 \pm 2.3 \approx)$.

\section{Stretch reflex}

In a sitting position, mechanically evoked stretch reflexes in SOL $\left(\mathrm{t}_{0}: 1.04 \pm 0.29 ; \mathrm{t}_{1}: 1 ; \mathrm{t}_{3}: 0.70 \pm 0.28 ; \mathrm{t}_{4}\right.$ :
$0.86 \pm 0.28$; and $t_{5}: 1.01 \pm 0.27, P<0.05$; Fig. 4 ) were significantly reduced immediately after WBV ( $\mathrm{t}_{3}$ : $P<0.05)$ compared with those established during the control condition before WBV. After WBV, a successive recovery of the stretch reflex amplitudes could be observed, and after $10 \mathrm{~min}$, the amplitudes reached the initial values established during the control condition before WBV (Fig. 5(a)).

Stretch reflex amplitudes in GM were significantly reduced immediately after compared with those established during the control condition before WBV ( $\mathrm{t}_{3}$ : $P<0.05$ ). However, $5 \mathrm{~min}$ and $10 \mathrm{~min}$ after $\mathrm{WBV}$, no statistically significant differences could be observed (for details, see Table 1 and Fig. 5(c)).

In a standing position, stretch reflex amplitudes in SOL (Fig. 5(b)) were decreased in trials after WBV compared with amplitudes established during the control condition before WBV $\left(\mathrm{t}_{0}: 1.06 \pm 0.32 ; \mathrm{t}_{1:} 1 ; \mathrm{t}_{3}\right.$ : $0.89 \pm 0.34 ; \mathrm{t}_{4}: 0.87 \pm 0.19 ; \quad$ and $\mathrm{t}_{5}: 0.87 \pm 0.26$, $P<0.05)$. GM showed a slight reduction in peak-to-peak amplitude in the trials after WBV compared with the amplitudes recorded during the control condition before vibration (for details, see Table 1 and Fig. 5(d)). However, the statistical analysis for stretch reflex amplitudes in GM revealed no significant effects $(P=1.00)$ in the standing position (Table 1).

\section{SLR in hopping}

No vibration-induced effects could be observed in the SLR during hopping in the SOL $\left(\mathrm{t}_{1}: 1 ; \mathrm{t}_{3}: 1.02 \pm 0.18 ; \mathrm{t}_{4}\right.$ : $1.07 \pm 0.21 ;$ and $\left.\mathrm{t}_{5}: 1.08 \pm 0.22, P=1.00\right), \mathrm{GM}\left(\mathrm{t}_{1}: 1 ; \mathrm{t}_{3}\right.$ : 


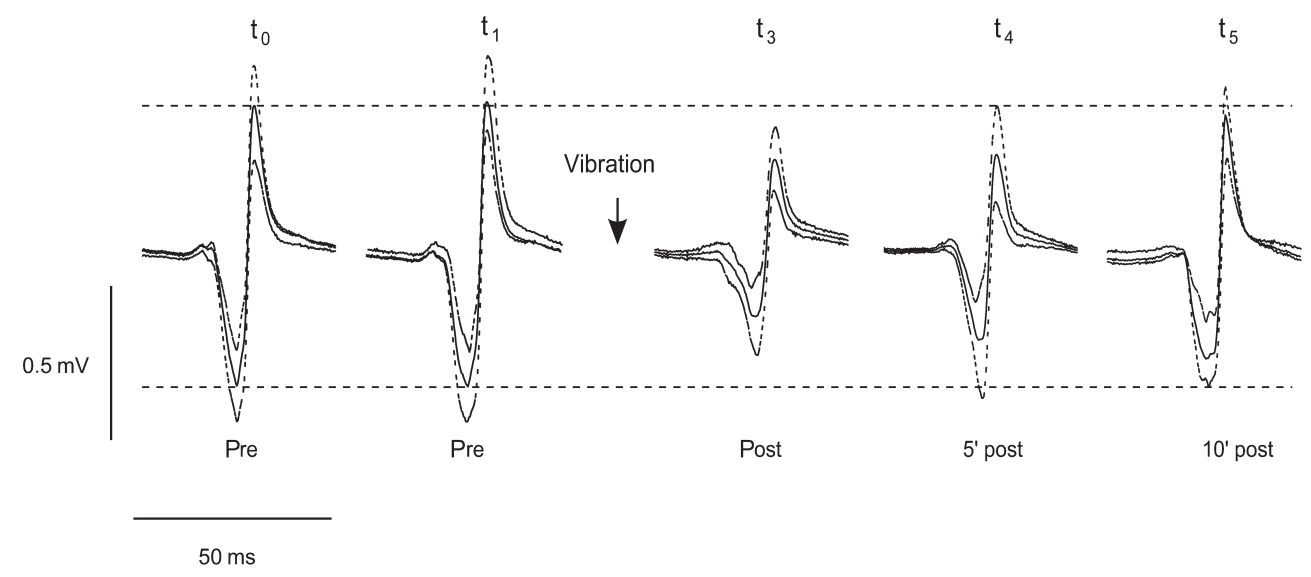

Fig. 4. Modulation of mechanically evoked stretch reflexes in the soleus muscle in response to acute WBV in one representative subject: mean and standard deviation of $2 \times 20$ stretch reflex responses recorded before $\left(t_{0}\right.$ and $\left.t_{1}\right)$, immediately after $\left(t_{3}\right), 5$ min $\left(t_{4}\right)$ and 10 min $\left(t_{5}\right)$ after vibration treatment. Stretch reflex amplitudes recorded before WBV displayed in $t_{0}$ and $t_{1}$ are very similar, whereas after WBV stretch reflex, amplitudes were reduced up to 5 min after WBV but had recovered 10 min afterwards.

M. soleus sitting

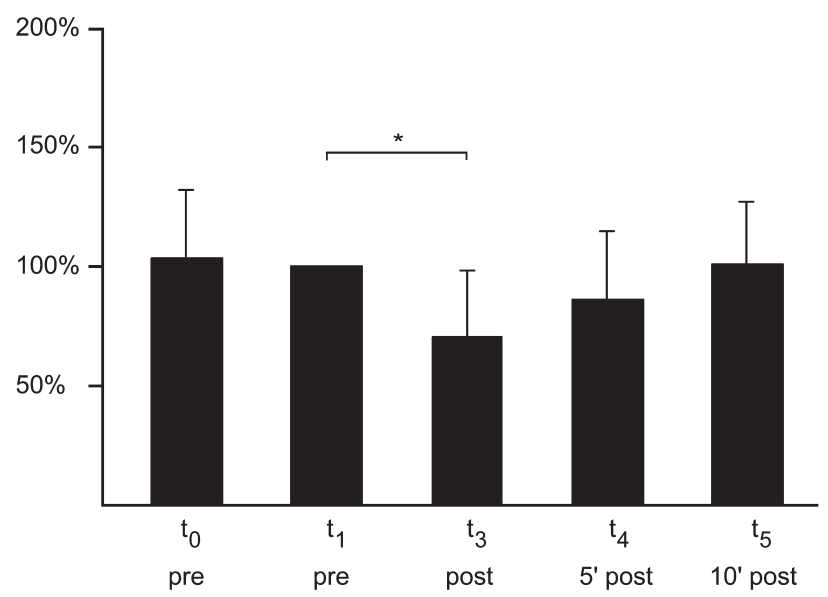

c M. gastrocnemius medialis sitting

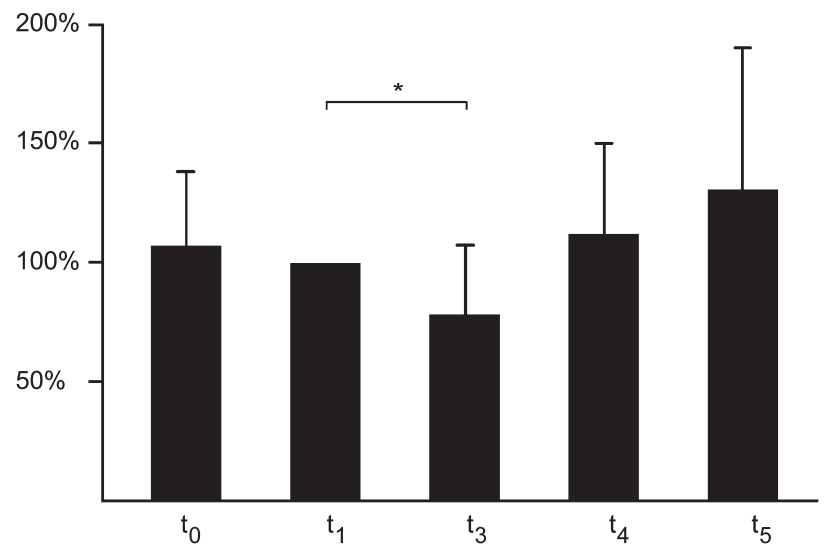

b

M. soleus standing

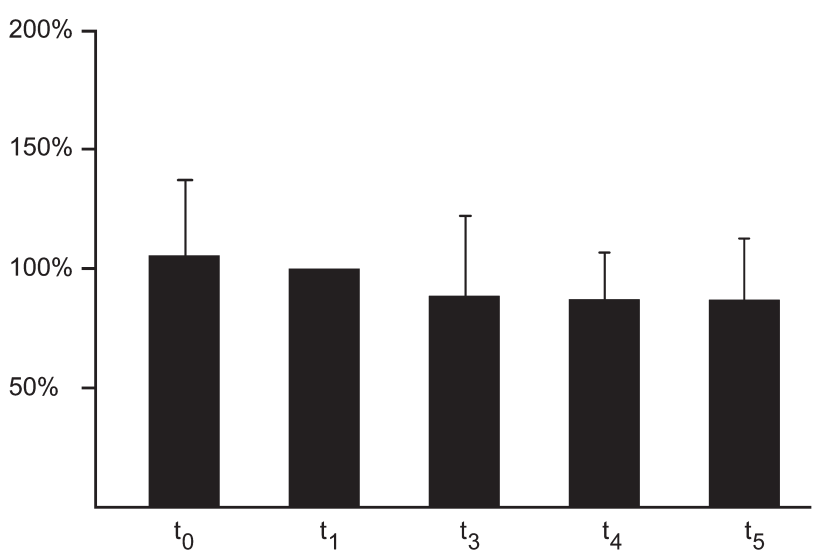

d

M. gastrocnemius medialis standing

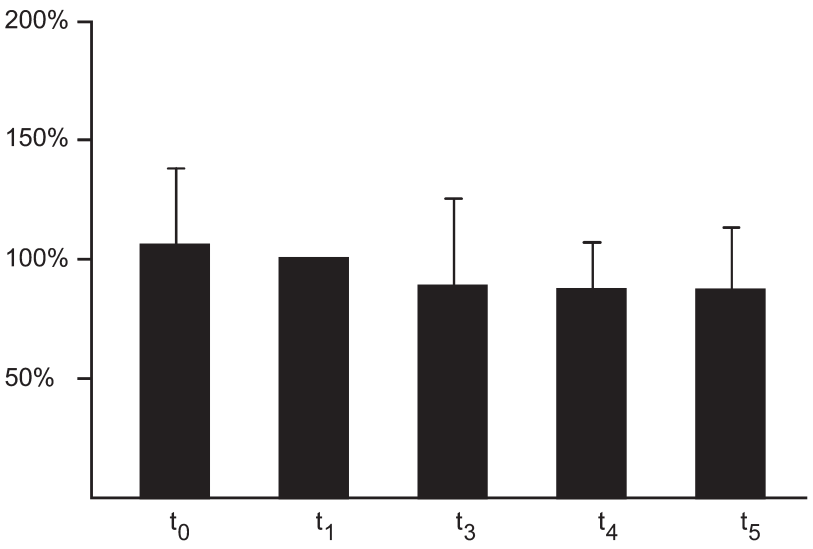

Fig. 5. Grand mean of stretch reflex amplitudes at the different time intervals before $\left(\mathrm{t}_{0}\right.$ and $\left.\mathrm{t}_{1}\right)$, immediately after $\left(\mathrm{t}_{3}\right), 5 \mathrm{~min}$ and $10 \mathrm{~min}$ after vibration treatment $\left(t_{4}\right.$ and $\left.t_{5}\right)$ in two modalities: in the soleus muscle during sitting (a) and standing (b), in the gastrocnemius medialis muscle during sitting (c) and standing (d). The stretch reflex amplitudes in the control conditions $\left(\mathrm{t}_{0}\right.$ and $\left.\mathrm{t}_{1}\right)$ were of similar size, whereas immediately after vibration treatment amplitudes were reduced $\left(\mathrm{t}_{3}\right)$. 
Table 1. Changes in stretch reflex amplitudes in the gastrocnemius medialis muscle

\begin{tabular}{lllllll}
\hline Stretch reflex & $\mathrm{t}_{0}$ & $\mathrm{t}_{1}$ & $\mathrm{t}_{3}$ & $\mathrm{t}_{4}$ & $\mathrm{t}_{5}$ & ANOVA results \\
\hline Sitting & $1.07 \pm 0.31$ & $1.00 \pm 0$ & $0.78 \pm 0.30$ & $1.12 \pm 0.39$ & $1.31 \pm 0.66$ & $P<0.05$ \\
Standing & $1.08 \pm 0.30$ & $1.00 \pm 0$ & $0.93 \pm 0.34$ & $0.95 \pm 0.19$ & $0.90 \pm 0.23$ & $P=1.00$ \\
\hline
\end{tabular}

Mean stretch reflex amplitudes of the gastrocnemius medialis muscle (normalized to $t_{1}$ ) evoked in the ankle ergometer in the sitting and standing position at the time intervals before $\left(t_{0}\right.$ and $\left.t_{1}\right)$, immediately after $\left(t_{3}\right), 5$ min and 10 min after WBV treatment $\left(t_{4}\right.$ and $\left.t_{5}\right)$.

ANOVA, analysis of variance; t, time intervals.

Table 2. Electromyographic data of hopping

\begin{tabular}{llllll}
\hline & $\mathrm{t}_{1}$ & $\mathrm{t}_{3}$ & $\mathrm{t}_{4}$ & $\mathrm{t}_{5}$ & ES results \\
\hline Pre SOL & $1.00 \pm 0$ & $1.02 \pm 0.07^{\approx}$ & $1.11 \pm 0.19^{\sim}$ & $1.13 \pm 0.24^{\approx}$ & Statistically equal \\
Pre GM & $1.00 \pm 0$ & $1.02 \pm 0.14^{\approx}$ & $1.02 \pm 0.07^{\sim}$ & $1.02 \pm 0.09^{\sim}$ & Statistically equal \\
Pre TA & $1.00 \pm 0$ & $1.03 \pm 0.12^{\approx}$ & $1.07 \pm 0.13^{\approx}$ & $1.03 \pm 0.14^{\approx}$ & Statistically equal \\
SLR TA & $1.00 \pm 0$ & $0.96 \pm 0.23$ & $0.97 \pm 0.20^{\approx}$ & $0.88 \pm 0.19$ &
\end{tabular}

Means and standard deviations of the soleus (SOL), gastrocnemius medialis (GM), and tibialis anterior muscles (TA) during the phase of preactivation (Pre) and for TA during the phase of the short-latency response (SLR) pre- $\left(t_{1}\right)$ and post-WBV treatment $\left(t_{3}, t_{4}\right.$, and $\left.t_{5}\right)$. Data are normalized to $t_{1}$. Parameters marked with $\approx$ were statistically equal.

ES, equivalence statistics; t, time intervals.

Table 3. Mechanical data of hopping

\begin{tabular}{lccccc}
\hline & $\mathrm{t}_{1}$ & $\mathrm{t}_{3}$ & $\mathrm{t}_{4}$ & $\mathrm{t}_{5}$ & ES results \\
\hline $\mathrm{F}_{\max }(\mathrm{N})$ & $3190 \pm 685$ & $3147 \pm 661^{\sim}$ & $3138 \pm 558^{\approx}$ & $3141 \pm 641^{\approx}$ & Statistically equal \\
GCT $(\mathrm{ms})$ & $188 \pm 33$ & $187 \pm 29^{\sim}$ & $184 \pm 26^{\approx}$ & $187 \pm 27^{\approx}$ & Statistically equal \\
RFD $(\mathrm{kN} / \mathrm{s})$ & $20.3 \pm 6.1$ & $20.4 \pm 6.1^{\approx}$ & $20.4 \pm 6.1^{\approx}$ & $20.4 \pm 5.8^{\approx}$ & Statistically equal \\
Height $(\mathrm{cm})$ & $17 \pm 3$ & $17 \pm 4^{\approx}$ & $16 \pm 3^{\approx}$ & $17 \pm 3^{\approx}$ & Statistically equal
\end{tabular}

Means and standard deviations of the maximal force $\left(F_{\max }\right)$, ground contact time $(\mathrm{GCT})$, rate of force development (RFD), and jump height of the hopping pre- $\left(t_{1}\right)$ and post-WBV treatment $\left(t_{3}, t_{4}\right.$, and $\left.t_{5}\right)$. Parameters marked with $\approx$ were statistically equal.

$E S$, equivalence statistics; $t$, time intervals.

$1.00 \pm 0.06 ; \quad \mathrm{t}_{4}: 1.04 \pm 0.13 ;$ and $\mathrm{t}_{5}: 1.02 \pm 0.10$ $P=1.00$ ), and TA muscles (Table 2). The EMG activity during the phase of preactivation in SOL, GM, and TA did not change in response to WBV and was statistically equal (for details, see Table 2). $F_{\max }$, RFD, GCT, and jump height of the hopping are displayed in Table 3; they were statistically equal.

\section{Discussion}

The purpose of this study was to ascertain the effects of acute WBV at specific times during and after the treatment on reflex responses on different complexity levels: the electrically elicited H-reflex, which bypasses the muscle spindles, the mechanically evoked stretch reflex, and the SLR during hopping, where spinal stretch reflexes interact with centrally programmed motor commands (Zuur et al., 2010). The main findings were that (1) the H-reflex in SOL was reduced during WBV. This reduction lasted for at least $5 \mathrm{~min}$. (2) WBV decreased the stretch reflex response in SOL and GM; however, the depression lasted at least for $1 \mathrm{~min}$ but less than $5 \mathrm{~min}$. (3) The SLR during hopping remained unaffected by WBV.

Thus, the current study demonstrated for the first time in a systematic and concise way that WBV interventions have a suppressive effect on spinal reflex responses when measurements were performed under rest conditions. However, the more active the test situation was, the less pronounced was the reflex inhibition resulting in no observable suppression during hopping. Apart from the test situation, the second main effect was the time when the reflexes were elicited. The strongest suppression could be monitored during WBV. Thereafter, the suppressive effect declined over time. Importantly, no significant reflex facilitation could be observed at any time in any of the tested situations. This seems of great relevance as previous studies speculated that some of the performance improvements observed after WBV occurred because of an increased reflex contribution (Bosco et al., 2000; Cochrane \& Stannard, 2005). Based on the present results, which are well in line with obser- 
vations obtained after locally applied tendon and muscle vibrations (Gail et al., 1966; van Boxtel, 1986), it seems unlikely that changes within the central nervous system augment reflex responses because of WBV.

Previously, Armstrong et al. (2008) and Sayenko et al. (2010) reported a depression of the SOL H-reflex immediately after WBV. The present results confirmed this observation and further specified that the H-reflex depression was most pronounced during WBV and recovered slowly afterwards but was still not fully recovered $5 \mathrm{~min}$ after WBV. The potential mechanisms responsible for the reduction of the Ia afferent transmission are manifold and can occur through a variety of mechanisms: a $22 \mathrm{~Hz}$ WBV - as it was used in this study - results in 22 repetitive reflex-induced activations of the muscles per second (Ritzmann et al., 2010). Thus, the reduction of the H-reflex during WBV could be explained by post activation depression. Post activation depression reflects a reduced Ia afferent transmitter release because of previous activation (Crone \& Nielsen, 1989). The repetitive excitations cause a transmitter depletion within the presynaptic terminals and consequently a reduced postsynaptic excitation (Pinco \& Lev-Tov, 1993). In every cycle, WBV generates a dorsiflexion moment, stretches the calf muscles (Cochrane et al., 2009), and thus induces a successive and frequency-dependent activation of the muscle spindles (Ritzmann et al., 2010). During WBV, this excitation is transmitted via Ia afferents and generates numerous discharges at the $\alpha$-motoneuron pool (Cochrane et al., 2009; Ritzmann et al., 2010). Therefore, post activation depression most likely contributes to the reduction of the H-reflex during and shortly after WBV. However, as post activation depression was documented to last for only $10 \mathrm{~s}$ (Crone \& Nielsen, 1989), it cannot explain the incomplete recovery of the H-reflex observed 5 min after WBV. Based on studies investigating the effects of locally applied tendon vibration, it may be speculated that PSI has also contributed to the observed H-reflex reduction. Several studies reported inhibited $\mathrm{H}$ - and stretch reflex responses during and after tendon vibration (Arcangel et al., 1971; van Boxtel, 1986), and it was demonstrated that this reduction was due to an increase in PSI elicited by GABAergic interneurons (Gillies et al., 1969). Reciprocal inhibition is another mechanism that could potentially have reduced the reflex responses in this study. However, as WBV was shown to cause a co-contraction (Berschin \& Sommer, 2004) and reciprocal inhibition is considered to be reduced during co-contraction (Nielsen \& Kagamihara, 1992), it seems rather unlikely that this mechanism may have suppressed the Ia afferent transmission.

Independent of the detailed knowledge about the neural processes responsible for the H-reflex depression, it is reasonable to assume that the same inhibitory mechanisms acting on the H-reflex also influence the stretch reflex, as both use the same pathways. Thus, post activation depression and PSI could also explain the reduction of the stretch reflexes in SOL and GM when measured in the sitting position. In contrast to the H-reflexes, stretch reflexes are dependent on the activity of the $\gamma$-motoneurons and can be modulated by the fusimotor drive, altering the threshold for the muscle spindle activation (Hagbarth \& Macefield, 1995).

In contrast to the H-reflex suppression, (1) the stretch reflex inhibition recovered much faster and (2) was not noticeable in the standing position. A possible explanation for (1) has been proposed by Morita et al. (1998). They stated that the discrepancy between the $\mathrm{H}$ - and stretch reflex is based on the different processing at the spinal level. They proposed that the excitation by means of PNS in the H-reflex causes just one synchronized activation, whereas the excitation of the primary muscle spindle endings results in a more dispersed volley (Morita et al., 1998). In addition, PSI induced by locally applied tendon vibration was shown to diminish the H-reflex to a greater extent than the stretch reflex (van Boxtel, 1986). Thus, based on both observations, it might be speculated that the higher sensitivity of the H-reflex toward PSI is responsible for the more pronounced and longer lasting depression of the H-reflex after WBV.

Concerning (2), it is well-known that the level of background activation (Butler et al., 1993) is a factor that could potentially explain the differences between the standing and the sitting position in stretch reflex sensitivity. Based on previous studies showing stronger reflex modulations during rest than in the active state, it might be assumed that inhibitory effects in response to WBV are masked in the activated muscle (Burke et al., 1992). Gollhofer and Rapp (1993) showed that the stretch reflex responses recovered fast following mechanical stimulation in the standing position when the triceps surae muscle was activated, whereas in the relaxed sitting position, the reflexes remained suppressed. They proposed that these differences may be caused by alterations in the $\alpha-\gamma$ linkage (Gollhofer \& Rapp, 1993), possibly due to a faster reformation of previously disrupted cross-bridge links in the intrafusal muscle fibers in a standing position (Gottlieb et al., 1981). Based on these studies, it may be argued that the stretch reflexes after WBV recovered faster in the standing position because of the enhanced functional relevance of stretch reflexes for controlling an upright body position (Gollhofer \& Rapp, 1993).

Furthermore, from a functional point of view, it is noteworthy that reflex modulation in the even more dynamic task of hopping was missing completely. It might be speculated that the dynamic type of voluntary muscle activation accelerated the recovery of the reflex circuit because of its functional importance in regard to stiffness regulation in the stretch-shortening cycle (Komi, 2000). It may be supposed that supraspinal modulation could have compensated the reduction of the 
Ia afferent transmission. Although Taube et al. (2008) assumed that spinal mechanisms are predominantly present at the time of the SLR, a recent study highlighted that the motor cortex contributes to the SLR amplitude. Zuur et al. (2010) showed that the SLR consists of a stretch reflex component but also a cortical contribution. Thus, to generate appropriate muscle stiffness in the muscles encompassing the ankle joint in the stretch shortening-cycle, supraspinal centers might have compensated the reduced Ia afferent transmission during hopping.

In summary, in all the test conditions, either a reflex inhibition or insignificant changes in response to WBV could be observed. In any case, there was no evidence for any reflex facilitation. Thus, performance improvements after the application of WBV in sports training are not likely to be caused by spinal facilitation. Improved performance after WBV has therefore to be explained differently. One likely mechanism contributing to enhanced performance after WBV may be the previously discovered effect of WBV on muscle temperature (Cochrane et al., 2008, 2010). As the increase in performance was related to the increase in temperature, Cochrane et al. (2008) proposed that changes in muscle temperature may be primarily responsible for enhancing short-term explosive events after WBV.

Regarding therapy, the results of the present study could help to explain the beneficial effects of WBV in people suffering from exaggerated reflex activity. In hemiparetic patients, for instance, the WBV-induced reduction of Ia afferent transmission might help to reduce the abnormal muscle tone and reduce the co-contraction of their muscles. Furthermore, the partial and largely long duration of reflex suppression after WBV is probably helpful to facilitate voluntary motor actions in patients with a high muscle tone caused by exaggerated Ia afferent input (Ness \& Field-Fote, 2009).
This assumption is well in line with previous observations showing that WBV training enhances the walking ability and balance control in persons with spastic diplegia (Ahlborg et al., 2006) and improves mobility and muscle force in bilateral spastic cerebral palsy children (Stark et al., 2010). Those improvements were accompanied by a reduction of spasticity that might be associated with a suppression of Ia afferent transmission (Ahlborg et al., 2006).

\section{Perspectives}

This study demonstrated an extensive suppression of Ia afferent transmission combined with a delayed recovery in response to WBV. However, with an increase in motor complexity, the suppression was less pronounced, and the recovery was accelerated. During hopping, the condition with the highest complexity level, WBV did not affect the reflex component. The present results of reduced reflex activity after WBV are well in line with the effects observed after locally applied tendon and muscle vibrations. Possible mechanisms explaining the suppression of reflex responses after WBV are post activation depression, PSI, and changes in muscle spindle sensitivity. Furthermore, it is proposed that in tasks with a greater motor complexity, the inhibitory mechanisms may be compensated by supraspinal facilitating mechanisms leading to an accelerated reflex recovery in the voluntarily activated muscle.

Key words: electromyography, EMG, stretch-shortening cycle, peripheral nerve stimulation, training.

\section{Acknowledgement}

This study was partially funded by the Deutsche Gesellschaft für Luft- und Raumfahrt.

\section{References}

Ahlborg L, Andersson C, Julin P. Whole-body vibration training compared with resistance training: effect on spasticity, muscle strength and motor performance in adults with cerebral palsy. J Rehabil Med 2006: 38 (5): 302-308.

Arcangel CS, Johnston R, Bishop B. The Achilles tendon reflex and the $\mathrm{H}$-response during and after tendon vibration. Phys Ther 1971: 51 (8): 889-905.

Armstrong WJ, Nestle HN, Grinnell DC, Cole LD, van Gilder EL, Warren GS, Capizzi EA. The acute effect of whole-body vibration on the Hoffmann reflex. J Strength Cond Res 2008: 22 (2): 471-476.

Berschin G, Sommer H.

Vibrationstraining und Gelenkstabilität:
EMG-Untersuchungen zur Wirkung von Vibrationsfrequenz und Körperhaltung auf Muskelaktivierung und -koaktivierung. Deutsche Zeitschrift Sportmedizin 2004: 55 (6): 152-156. Borman PJ, Chatfield MJ, Damjanov I, Jackson P. Design and analysis of method equivalence studies. Anal Chem 2009: 81 (24): 9849-9857.

Bosco C, Iacovelli M, Tsarpela O, Cardinale M, Bonifazi M, Tihanyi J, Viru M, de Lorenzo A, Viru A. Hormonal responses to whole-body vibration in men. Eur J Appl Physiol 2000: 81 (6): 449-454.

van Boxtel A. Differential effects of low-frequency depression, vibration-induced inhibition, and posttetanic potentiation on H-reflexes and tendon jerks in the human soleus muscle. J Neurophysiol 1986: 55 (3): 551-568.

Burke D, Hagbarth KE, Löfstedt L, Wallin BG. The responses of human muscle spindle endings to vibration during isometric contraction. J Physiol 1976: 261 (3): 695-711.

Burke D, Gracies JM, Meunier S, Pierrot-Deseilligny E. Changes in presynaptic inhibition of afferents to propriospinal-like neurones in man during voluntary contractions. J Physiol (Lond) 1992: 449: 673-687.

Butler AJ, Yue G, Darling WG. Variations in soleus H-reflexes as a function of plantarflexion torque in man. Brain Res 1993: 632 (1-2): 95-104.

Cardinale M, Bosco C. The use of vibration as an exercise intervention. Exerc Sport Sci Rev 2003: 31 (1): 3-7. 
Cochrane DJ, Stannard SR. Acute whole body vibration training increases vertical jump and flexibility performance in elite female field hockey players. Br J Sports Med 2005: 39 (11): 860-865.

Cochrane DJ, Stannard SR, Sargeant AJ, Rittweger J. The rate of muscle temperature increase during acute whole-body vibration exercise. Eur J Appl Physiol 2008: 103 (4): 441-448.

Cochrane DJ, Loram ID, Stannard SR, Rittweger J. Changes in joint angle, muscle-tendon complex length, muscle contractile tissue displacement, and modulation of EMG activity during acute whole-body vibration. Muscle Nerve 2009: 40 (3): 420-429.

Cochrane DJ, Stannard SR, Firth EC, Rittweger J. Comparing muscle temperature during static and dynamic squatting with and without whole-body vibration. Clin Physiol Funct Imaging 2010: 30 (4): 223-229.

Crone C, Nielsen J. Methodological implications of the post activation depression of the soleus H-reflex in man. Exp Brain Res 1989: 78 (1): 28-32.

Delecluse C, Roelants M, Verschueren S. Strength increase after whole-body vibration compared with resistance training. Med Sci Sports Exerc 2003: 35 (6): 1033-1041.

Gail P, de Lance JW, Neilson PD. Differential effects on tonic and phasic reflex mechanisms produced by vibration of muscles in man. J Neurol Neurosurg Psychiatr 1966: 29 (1): $1-11$.

Gillies JD, Lance JW, Neilson PD, Tassinari CA. Presynaptic inhibition of the monosynaptic reflex by vibration. J Physiol (Lond) 1969: 205 (2): 329-339.

Gollhofer A, Rapp W. Recovery of stretch reflex responses following mechanical stimulation. Eur J Appl Physiol Occup Physiol 1993: 66 (5): 415-420.

Gottlieb GL, Agarwal GC, Jaeger RJ. Response to sudden torques about ankle in man. IV. A functional role of alpha-gamma linkage. J Neurophysiol 1981: 46 (1): 179-190.

Granit R. The functional role of the muscle spindles - facts and hypotheses. Brain 1975: 98 (4): 531-556.

Hagbarth KE, Macefield VG. The fusimotor system. Its role in fatigue. Adv Exp Med Biol 1995: 384: 259-270.

Hayashi R, Tako K, Tokuda T, Yanagisawa N. Comparison of amplitude of human soleus H-reflex during sitting and standing. Neurosci Res 1992: 13 (3): 227-233.

Hobara H, Kanosue K, Suzuki S. Changes in muscle activity with increase in leg stiffness during hopping. Neurosci Lett 2007: 418 (1): 55-59.

Hopkins JT, Fredericks D, Guyon PW, Parker S, Gage M, Feland JB, Hunter I. Whole body vibration does not potentiate the stretch reflex. Int J Sports Med 2009: 30 (2): 124-129.

Komi PV. Stretch-shortening cycle: a powerful model to study normal and fatigued muscle. J Biomech 2000: 33 (10): 1197-1206.

McBride JM, Nuzzo JL, Dayne AM, Israetel MA, Nieman DC, Triplett NT. Effect of an acute bout of whole body vibration exercise on muscle force output and motor neuron excitability. J Strength Cond Res 2010: 24 (1): 184-189.

Matthews PB. Reflex activation of the soleus muscle of the decerebrate cat by vibration. Nature 1966: 209 (5019): 204-205.

Melnyk M, Kofler B, Faist M, Hodapp M, Gollhofer A. Effect of a whole-body vibration session on knee stability. Int J Sports Med 2008: 29 (10): 839-844.

Morita H, Petersen N, Christensen LO, Sinkjaer T, Nielsen J. Sensitivity of $\mathrm{H}$-reflexes and stretch reflexes to presynaptic inhibition in humans. J Neurophysiol 1998: 80 (2): 610-620.

Ness LL, Field-Fote EC. Effect of whole-body vibration on quadriceps spasticity in individuals with spastic hypertonia due to spinal cord injury. Restor Neurol Neurosci 2009: 27 (6): 621-631.

Nielsen J, Kagamihara Y. The regulation of disynaptic reciprocal Ia inhibition during co-contraction of antagonistic muscles in man. J Physiol (Lond) 1992: 456: 373-391.

Nordlund MM, Thorstensson A. Strength training effects of whole-body vibration? Scand J Med Sci Sports 2007: 17 (1): 12-17.

Pinco M, Lev-Tov A. Modulation of monosynaptic excitation in the neonatal rat spinal cord. J Neurophysiol 1993: 70 (3): 1151-1158.

Rees SS, Murphy AJ, Watsford ML. Effects of whole-body vibration exercise on lower-extremity muscle strength and power in an older population: a randomized clinical trial. Phys Ther 2008: 88 (4): 462-470.

Rittweger J. Vibration as an exercise modality: how it may work, and what its potential might be. Eur J Appl Physiol 2010: 108 (5): 877-904.

Rittweger J, Beller G, Felsenberg D. Acute physiological effects of exhaustive whole-body vibration exercise in man. Clin Physiol 2000: 20 (2): 134-142.

Rittweger J, Mutschelknauss M, Felsenberg D. Acute changes in neuromuscular excitability after exhaustive whole body vibration exercise as compared to exhaustion by squatting exercise. Clin Physiol Funct Imaging 2003: 23 (2): 81-86.

Ritzmann R, Kramer A, Gruber M, Gollhofer A, Taube W. EMG activity during whole body vibration: motion artifacts or stretch reflexes? Eur J Appl Physiol 2010: 110 (1): 143-151.

Roelants M, Delecluse C, Verschueren SM. Whole-body-vibration training increases knee-extension strength and speed of movement in older women. J Am Geriatr Soc 2004: 52 (6): 901-908.

Roll JP, Martin B, Gauthier GM, Mussa Ivaldi F. Effects of whole-body vibration on spinal reflexes in man. Aviat Space Environ Med 1980: 51 (11): 1227-1233.

de Ruiter CJ, van der Linden RM, van der Zijden MJA, Hollander AP, de Haan A. Short-term effects of whole-body vibration on maximal voluntary isometric knee extensor force and rate of force rise. Eur J Appl Physiol 2003: 88 (4-5): 472-475.

Sayenko DG, Masani K, Alizadeh-Meghrazi M, Popovic MR, Craven BC. Acute effects of whole body vibration during passive standing on soleus H-reflex in subjects with and without spinal cord injury. Neurosci Lett 2010: 482 (1): 66-70.

Stark C, Nikopoulou-Smyrni P, Stabrey A, Semler O, Schoenau E. Effect of a new physiotherapy concept on bone mineral density, muscle force and gross motor function in children with bilateral cerebral palsy.

J Musculoskelet Neuronal Interact 2010: 10 (2): 151-158.

Taube W, Leukel C, Schubert M, Gruber M, Rantalainen T, Gollhofer A. Differential modulation of spinal and corticospinal excitability during drop jumps. J Neurophysiol 2008: 99 (3): 1243-1252.

Zuur AT, Lundbye-Jensen J, Leukel C, Taube W, Grey MJ, Gollhofer A, Nielsen JB, Gruber M. Contribution of afferent feedback and descending drive to human hopping. J Physiol (Lond) 2010: 588 (Pt 5): 799-807. 